\title{
NOS3 Intron 4 VNTR
}

National Cancer Institute

\section{Source}

National Cancer Institute. NOS3 Intron 4 VNTR. NCI Thesaurus. Code C129419.

A repetitive sequence found in intron 4 of the NOS3 gene. 